\title{
Anisotropic superconductivity induced by periodic multiferroic domain patterns
}

\author{
Yen-Lin Huang', Bo-Chao Huang ${ }^{2}$, Yan-Ting Wang ${ }^{3}$, Fei Xue ${ }^{4}$, Jheng-Cyuan Lin², Heng-Jui Liu (1) ${ }^{5}$, Rong Huang ${ }^{6,7}$,

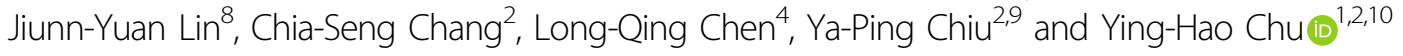

\begin{abstract}
The competition between order parameters, such as ferroelectricity, ferromagnetism, and superconductivity, is one of the most fascinating topics in condensed matter physics. Here, we report intriguing anisotropic superconductivity in $\mathrm{YBa}_{2} \mathrm{Cu}_{3} \mathrm{O}_{7-x}$ films induced by a multiferroic, $\mathrm{BiFeO}_{3}$, with periodic domain patterns. The anisotropic superconductivity was investigated by transport measurements and supported by phase-field simulations, and then the detailed local electronic structures were revealed by cross-sectional scanning tunneling microscopy. We found that the oxygen redistribution in $\mathrm{YBa}_{2} \mathrm{Cu}_{3} \mathrm{O}_{7-x}$ modulated by the ferroelectric polarization in $\mathrm{BiFeO}_{3}$ was the key mechanism driving this anisotropic superconductivity. The presented heteroarchitecture of a high-temperature superconductor and a domain-engineered multiferroic provides a new approach to tune superconductivity and offers potential advantages for the design of future multifunctional devices.
\end{abstract}

\section{Introduction}

The reversible switching ability of order parameters is a distinguishing key feature of spontaneous ordering in ferroics. Among the numerous ferroic systems that have been explored, multiferroics have recently aroused great scientific interest due to the coexistence and intriguing coupling between order parameters, allowing the modulation of one through another ${ }^{1-5} \cdot \mathrm{BiFeO}_{3}$ (BFO) has played an important role in this field after the discovery of its large ferroelectric polarization $\left(\sim 100 \mu \mathrm{C} / \mathrm{cm}^{2}\right)^{6}$ and multiferroic characteristics above room temperature ${ }^{7,8}$, making it appealing for practical applications ${ }^{9}$. Perovskite $\mathrm{BFO}$ possesses the space group of $R 3 c$ with spontaneous ferroelectric polarization along pseudocubic <111> directions and in the G-type antiferromagnetic ordering; as a result of the free energy competition, the ferroics form domains ${ }^{9,10}$. In BFO, three different angles $\left(71^{\circ}\right.$, $109^{\circ}$, and $180^{\circ}$ ) can be found between the ferroelectric

\footnotetext{
Correspondence: Ying-Hao Chu (yhchu@g2.nctu.edu.tw)

'Department of Materials Science and Engineering, National Chiao Tung

University, Hsinchu, Taiwan

${ }^{2}$ Institute of Physics, Academia Sinica, Taipei, Taiwan

Full list of author information is available at the end of the article
}

polarization in adjacent domains. In addition, domain walls in the BFO possess rich physical properties, such as magnetoresistance and exchange bias ${ }^{11,12}$. Recently, progress in the domain engineering of BFO has been extensive, leading to the control of periodic domain patterns with long-range ordering, which can be used for the both fundamental research and the exploration of potential applications.

Moreover, the tangled ferroic orders in multiferroics result in the ability to provide a unique environment to the heterostructures via various coupling mechanisms. By precisely controlling domain patterns, a ferroic system can improve the electric, magnetic, and structural coupling to adjacent layers. For example, studies performed by Heron et al. ${ }^{13,14}$ and Trassin et al. ${ }^{15}$ show an excellent demonstration of the electrical control of ferromagnetism with the $\mathrm{Co}_{0.9} \mathrm{Fe}_{0.1} / \mathrm{BFO}$ heterostructure via a one-to-one correlation of the ferroelectric in the $\mathrm{BFO}$ and ferromagnetic domains in the $\mathrm{Co}_{0.9} \mathrm{Fe}_{0.1}$. In addition, uniaxial magnetic anisotropy and anisotropic magnetoresistance have been observed in a $\mathrm{La}_{0.7} \mathrm{Sr}_{0.3} \mathrm{MnO}_{3}$ layer grown on a periodic $71^{\circ} \mathrm{BFO}$ domain pattern, where the magnetic easy axis of the $\mathrm{La}_{0.7} \mathrm{Sr}_{0.3} \mathrm{MnO}_{3}$ and ferroelectric 
polarization of the BFO show parallel coupling via structural coupling ${ }^{16,17}$. Thus, the utilization of periodic multiferroic domain patterns can be a way to trigger very intriguing phenomena. In this study, a model system based on a combination of the high- $T_{\mathrm{c}}$ superconductor $\mathrm{YBa}_{2} \mathrm{Cu}_{3} \mathrm{O}_{7-x}(\mathrm{YBCO})$ and multiferroic $\mathrm{BFO}$ with carefully designed domain configurations are introduced. The superconductivity in the YBCO layer was thoroughly explored upon undergoing long-range-ordered electrostatic, elastic, and antiferromagnetic perturbations provided by neighboring multiferroic BFO domain patterns. Surprisingly, anisotropic superconductivity was discovered in the YBCO layer. The insights of this emerging phenomenon were unveiled by cross-sectional scanning tunneling microscopy (STM) and supported by phasefield simulations. Such a result provides an elegant approach to tune the local superconductivity of a superconductor, which has great potential to trigger additional device applications based on high-temperature superconductors.

\section{Materials and methods}

Thin film growth and characterization

YBCO/BFO films with thicknesses between 15 and $180 \mathrm{~nm}$ were grown on single-crystal $(110)_{\mathrm{O}} \mathrm{DyScO}_{3}$ (DSO) substrates by pulsed laser deposition from $650-700{ }^{\circ} \mathrm{C}$ at an oxygen pressure of 100 mTorr. The samples were cooled at a rate of $20^{\circ} \mathrm{C} / \mathrm{min}$ at an oxygen pressure of 600 Torr and dwelled at $500{ }^{\circ} \mathrm{C}$ for $60 \mathrm{~min}$. The typical deposition rate was $\sim 2 \mathrm{~nm} / \mathrm{min}$ with a laser repetition rate of $10 \mathrm{~Hz}$. The focused laser power density on the target surface was $\sim 2 \mathrm{~J} / \mathrm{cm}^{2}$. The structural details of the samples were studied by synchrotron-based X-ray diffraction techniques. The RSMs of the heterostructures were performed using a PANalytical X'Pert Pro 4-circle X-ray diffractometer with $\mathrm{Cu} \mathrm{K \alpha} \alpha_{1}$ radiation $(\lambda=1.54 \AA$ ), recorded with a series of $\theta-2 \theta$ scans with different $\omega$ offsets and collected at beamline BL-17B1 at the National Synchrotron Radiation Research Center in Hsinchu, Taiwan.

The ferroelectric domain structures were characterized at room temperature by piezoresponse force microscopy (PFM, Veeco Multimode). The tips used for the PFM imaging were Ti-Pt-coated cantilevers with an elastic constant of $4.5 \mathrm{~N} / \mathrm{m}$ and a resonance frequency of $120-190 \mathrm{kHz}$.

\section{Transport measurement}

The transport measurement was performed in a physical property measurement system (Quantum Design). The resistance measurements were obtained during both heating and cooling paths with an applied current of $1 \mu \mathrm{A}$ within a temperature range from 300 to $2 \mathrm{~K}$ with a cooling rate of $1 \mathrm{~K} / \mathrm{min}$.

\section{Scanning tunneling microscopy}

The experiments used DSO as the substrates for the STM study. A 30-nm BFO layer was deposited prior to the growth of the $35-45 \mathrm{~nm}$ YBCO layers. Finally, a $500 \mathrm{~nm}$ amorphous Al-doped $\mathrm{ZnO}$ (AZO) buffer layer was deposited on the YBCO to prevent a tip crash during the STM measurements. The samples were cooled down to $25 \mathrm{~K}$ and then cleaved in situ in an ultrahigh vacuum chamber with a base pressure of $5 \times 10^{-11}$ Torr. All STM measurements were performed at $25 \mathrm{~K}$.

\section{Results and discussion}

In the rhombohedrally distorted perovskite $\mathrm{BFO}$, there are eight possible directions for ferroelectric polarization that point along the pseudocubic $<111>$ directions, corresponding to four structural variants. The formation and control of ferroelectric domain patterns in (001)-oriented rhombohedral perovskite films have been well established in earlier theoretical and experimental studies ${ }^{18-20}$. The heteroepitaxial constraints can lead to periodic nanoscale arrays of ferroelastic domains, such as the $109^{\circ}$ and $71^{\circ}$ domain configurations in BFO films. The same approach was adopted in this study with the $\mathrm{DyScO}_{3}(110)_{\mathrm{O}}$ (DSO, where "O" denotes the orthorhombic system) substrates, and the detailed growth process can be found in our previous work $^{20}$ and the Methods section herein. PFM was employed to reveal the nicely ordered $109^{\circ}$ and $71^{\circ}$ domain patterns (Fig. 1a, d, respectively). We note that the ferroelectric polarization configurations are different in the $109^{\circ}$ and $71^{\circ}$ patterns shown in Fig. 1b, e. In the $109^{\circ}$ pattern, the out-of-plane polarizations are periodically pointing up and down; on the other hand, in the $71^{\circ}$ pattern, the out-of-plane polarizations are uniformly pointing down. Detailed PFM images of both domain patterns are shown in S1 in the Supplementary Materials. The YBCO layers were deposited on these BFO films to form heteroepitaxial systems. The structural information of the heterostructures was extracted from a series of X-ray diffraction reciprocal space maps (RSMs) shown in Fig. 1c, f. The normal scan (00L) shows a high-quality, single-phase, and $c$-oriented YBCO and BFO layer in S2. For the underlying BFO films, the typical diffraction characteristics of $109^{\circ}$ and $71^{\circ}$ domain patterns can be observed in the asymmetric RSMs along the DSO $(222)_{\mathrm{O}}$ reflections. For the $109^{\circ}$ domain pattern, the normal vector of the atomic planes in the $109^{\circ}$ domain pattern has an angle difference $\left(\sim 0.68^{\circ}\right)$ between the neighbor domains ${ }^{20,21}$. Thus, we can observe a horizontal splitting of the $(012)_{\mathrm{pc}}$ reflection (where "pc" denotes the pseudocubic system) (Fig. 1c) with the $109^{\circ}$ domain pattern, whereas the peak splitting cannot be observed for the same reflection in the $71^{\circ}$ domain pattern (Fig. 1f). On the other hand, for the $71^{\circ}$ domain pattern, the normal vector of the atomic planes and lattice constant are the same 
(a)

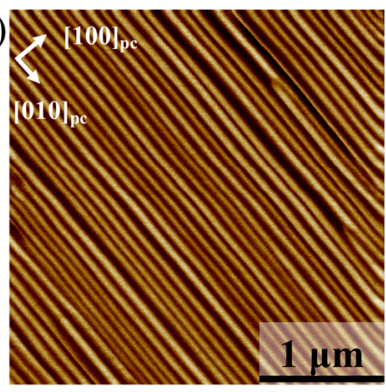

(d)

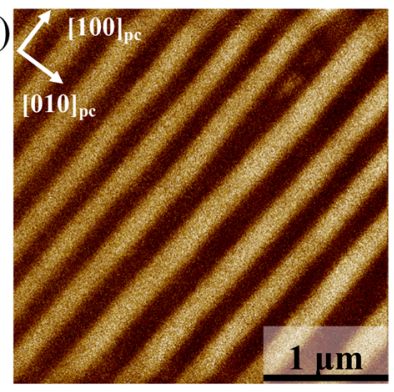

(b)

\section{$109^{\circ} \mathrm{DW}$}
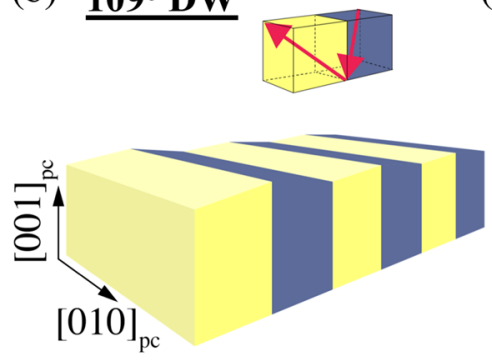

(e)

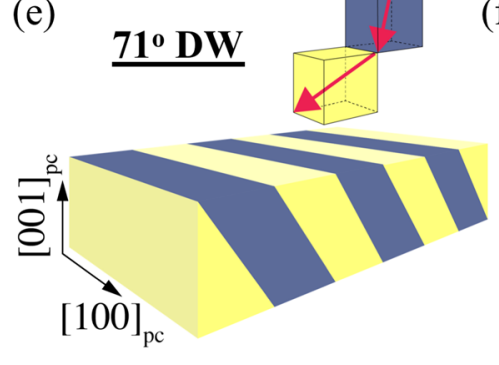

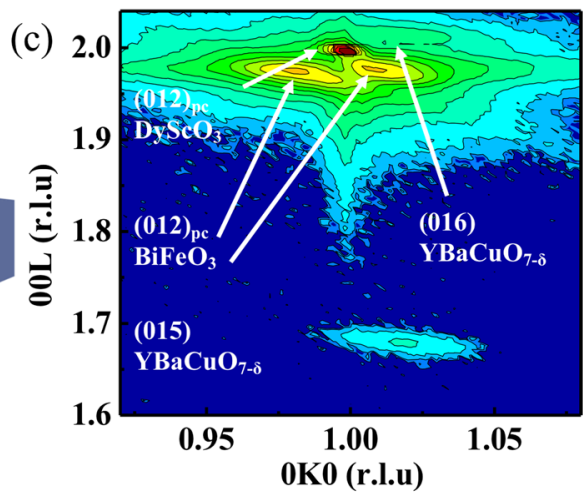

(f)

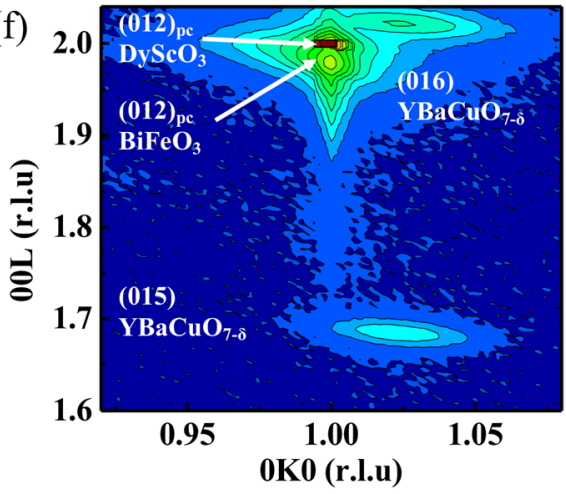

Fig. 1 Model domain-wall architecture. a, d PFM images of ordered arrays for $109^{\circ}$ and $71^{\circ}$ domain patterns, respectively. $\mathbf{b}$, e Schematic of the heterostructure of the $109^{\circ}$ and $71^{\circ}$ domain-wall arrays, respectively. $\mathbf{c}$, f Reciprocal space mapping of the (012) ${ }_{p c}$ peaks from BFO and DSO and $(016)_{p c}$ and $(015)_{p c}$ peaks from YBCO for $109^{\circ}$ and $71^{\circ}$ cases, respectively.

Table 1 Lattice parameter of the YBCO layer of $109^{\circ}$ and $71^{\circ}$ cases, respectively.

\begin{tabular}{llll}
\hline & $\boldsymbol{a}(\AA)$ & $\boldsymbol{b}(\AA)$ & $\boldsymbol{c}(\AA)$ \\
\hline $109^{\circ}$ case & $3.84(6)$ & $3.85(5)$ & $11.68(0)$ \\
$71^{\circ}$ case & $3.87(2)$ & $3.88(7)$ & $11.71(0)$ \\
\hline
\end{tabular}

between the domains, resulting in a single BFO peak, as shown in Fig. 1f. These measurements show that the BFO layer retained its domain structure after the consecutive deposition of the YBCO layer. The corresponding BFO lattice parameters were extracted and are presented in Table 1 of the supporting materials. In addition, the $\{105\}$ and $\{106\}$ YBCO diffraction peaks can also be observed in these RSMs. The YBCO layer maintained its orthorhombic structure (Fig. 1c, d) and possessed a monodomain structure instead of twinned structures, presenting an elongated $a$-axis compared to that of the bulk YBCO to achieve a coherent strain with the BFO film and DSO substrate. In bulk orthorhombic $\mathrm{YBCO}$, the $\mathrm{Cu}-$ $\mathrm{O}$ chains are typically formed along the $b$-axis between the $\mathrm{CuO}_{2}$ planes. These chains provide conducting channels for charge carriers, causing an anisotropic in-plane conductivity. Thus, the anisotropic lattice structure of $\mathrm{YBCO}$ can contribute to a low anisotropic transport behavior due to the direction of the $\mathrm{Cu}-\mathrm{O}$ chains. However, when the temperature was lower than the critical temperature $\left(T_{\mathrm{c}}\right)$, the $\mathrm{YBCO}$ only shows isotropic superconductivity ${ }^{22}$. Here, we stress that the YBCO layers in this study exhibit single-crystal features; therefore, we can exclude any anisotropy that may be induced by grain boundaries or the bicrystal effect ${ }^{23}$. We also note that the existence of vicinal steps or terraces in the BFO films should be excluded in this anisotropic superconductivity discussion because the terrace does not have a particular correlation with the direction the $71^{\circ}$ domain walls (shown in S3). The ultimate goal of this study is to create anisotropic superconductivity in the heterostructures of $\mathrm{YBCO} / \mathrm{BFO}$ by perturbing the pairing of Cooper pairs or modulating the doping level in the $\mathrm{YBCO}$ layer in only one direction via the long-range stripy domain patterns in the BFO layer at the nanoscale.

To explore the proposed anisotropic superconductivity, transport measurements were carried out on two sets of heterostructures: $\mathrm{YBCO} / \mathrm{BFO}$ with $109^{\circ}$ (denoted as the " $109^{\circ}$ case") and $71^{\circ}$ domain patterns (denoted as the " $71^{\circ}$ 


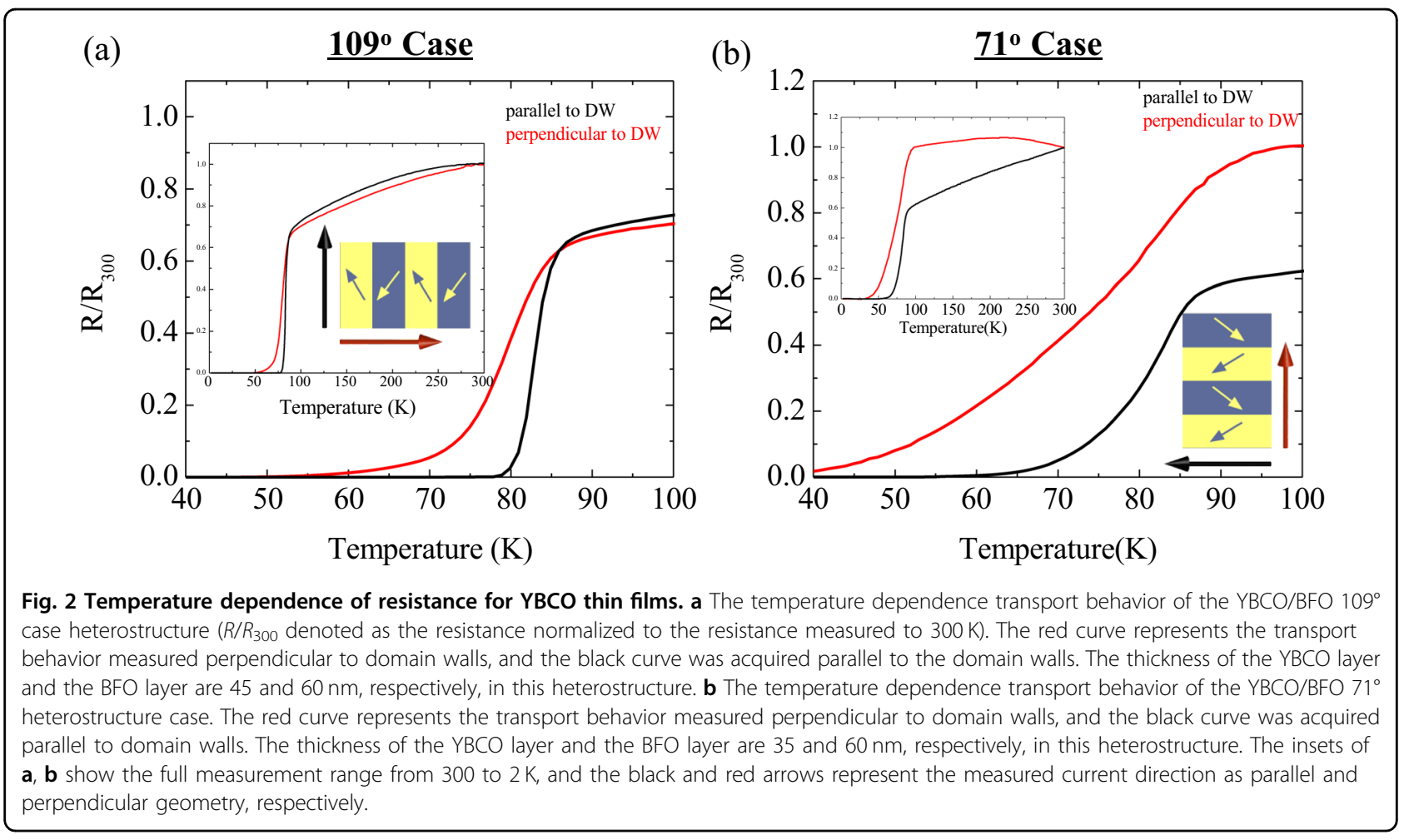

case"). The DC resistance of the YBCO layers was extracted from the voltage drop measured by a fourterminal apparatus with a $1 \mu \mathrm{A}$ current flowing parallel $\left([010]_{\mathrm{pc}}\right.$ in the $109^{\circ}$ case and $[100]_{\mathrm{pc}}$ in the $71^{\circ}$ case and denoted as a "parallel geometry," shown as the black arrow in the inset of Fig. 2a, b, respectively) and perpendicular $\left([100]_{\mathrm{pc}}\right.$ in the $109^{\circ}$ domain pattern and $[010]_{\mathrm{pc}}$ in the $71^{\circ}$ domain pattern and denoted as a "perpendicular geometry," shown as the red arrow in the inset of Fig. 2a, $\mathrm{b}$, respectively) to the domain walls in all samples. The temperature dependence of the resistance $(300-2 \mathrm{~K})$ is shown as the black and red curves for the two different measurement geometries in Fig. 2a, b with the data normalized to the resistance at $300 \mathrm{~K}$. The trend of the $R-T$ curves indicates that these YBCO films were underdoped $^{24,25}$. Interestingly, a large anisotropy with greater than a $20 \mathrm{~K}$ difference in the offset temperatures was found for the two measured geometries, which maintained similar onset temperatures $(\sim 80 \mathrm{~K})$. The anisotropic behavior discovered in these heterostructures is defined as an "easy axis" for superconductivity, where a direction with a higher $T_{\mathrm{c}}$ can always be observed. It is very intriguing to see that the easy axis for superconductivity in our system is always parallel to the direction of the domain walls in the BFO layer $\left([100]_{\mathrm{pc}}\right.$ in the $109^{\circ}$ case, and $[010]_{\mathrm{pc}}$ in the $71^{\circ}$ case), suggesting that the anisotropic superconductivity is strongly correlated to the domain patterns in the BFO layer.
One possible hypothesis is that the ferroelectric field effect from the BFO layer modulates the carrier distribution in the YBCO layer, resulting in an anisotropic distribution of the doping level in the $\mathrm{YBCO}^{26,27}$ during the growth process since BFO has a high Curie temperature of $\sim 1100 \mathrm{~K}$. The controlled YBCO thickness dependence transport measurements support an interface effect (shown in S4). The anisotropic superconductivity vanishes beyond $48 \mathrm{~nm}$ of the YBCO. This suggests that the magnetic proximity effect might not be the dominant factor due to the short-range nature of the exchange interaction, yet the oxygen redistribution or elastic relaxation might play a very important role in our case. These hypotheses are further verified by a combination of phase-field simulations and cross-sectional STM (XSTM).

The superconductivity of YBCO is very sensitive to the oxygen doping level, which suggests that the oxygen content in the YBCO film determines the $T_{\mathrm{c}}$. Phase-field simulations were employed to investigate the distribution of oxygen vacancies in the YBCO layer under the influence of periodic BFO domain patterns (see S5-S7 in the Supplementary Materials for details). In the simulation results, the $109^{\circ}$ domain patterns show a periodic configuration of upward and downward polarizations. Under the influence of the electrostatic potentials, the oxygen vacancies can diffuse at high temperatures during growth, leading to the redistribution of the oxygen in the YBCO layer, as shown in Fig. 3a. Such a redistribution of the 


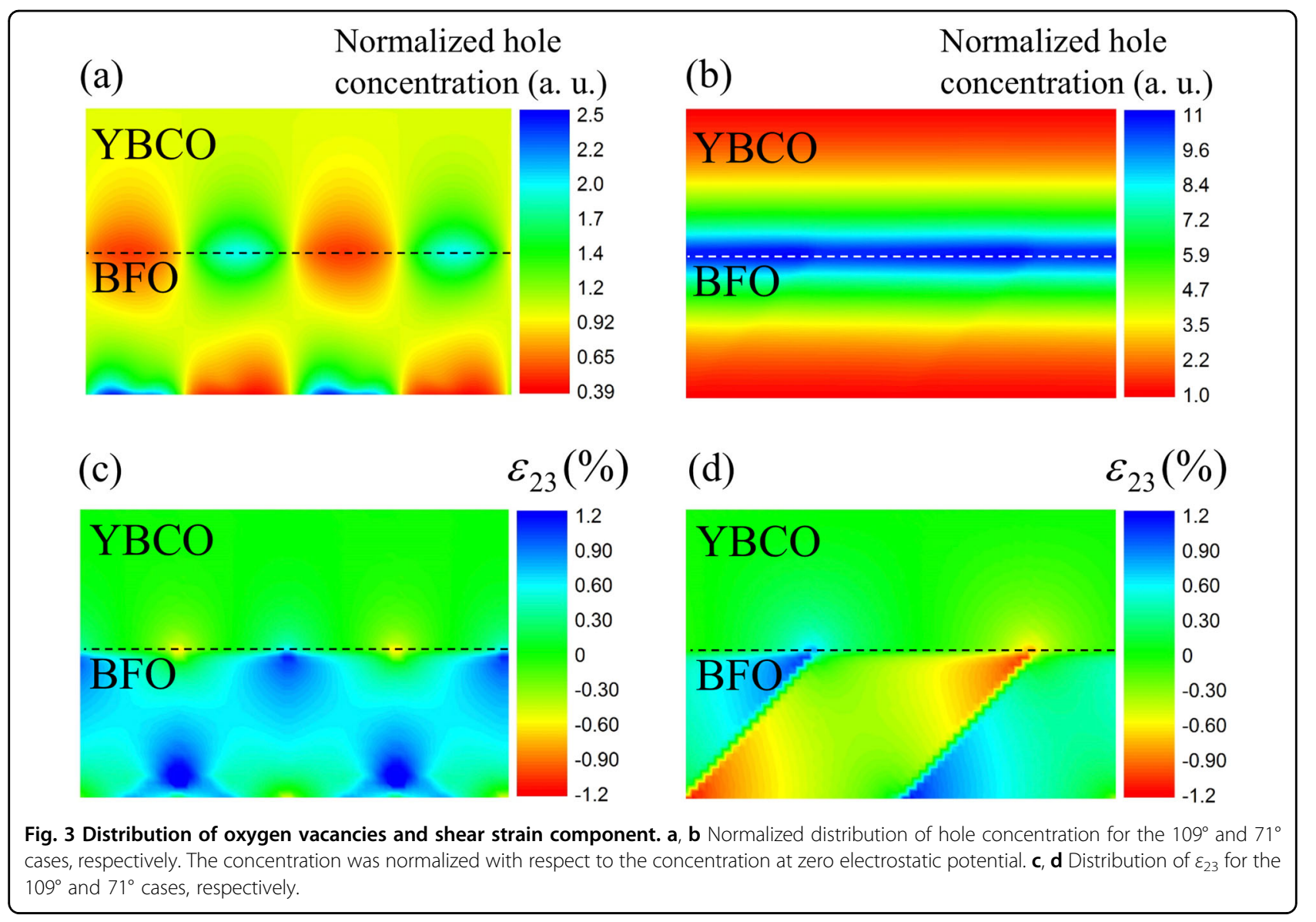

oxygen can result in different $T_{\mathrm{c}}$ in the YBCO regions correlated to the two domains in the BFO layer, which can be understood to consist of two parts with different resistances, namely a high- and low- $T_{\mathrm{c}}$ region. With the two types of regions connected in parallel, the superconductivity was determined by the high- $T_{\mathrm{c}}$ region for the parallel geometry (easy axis), whereas for the perpendicular geometry, the two types of regions are serially connected, and the overall $T_{\mathrm{c}}$ was determined by the low$T_{\mathrm{c}}$ region (hard axis). On the other hand, for the $71^{\circ}$ case, with a homogenous downward polarization in the BFO layer (see S1 in Supplementary Materials), the distribution of the oxygen vacancies is almost uniform along the inplane direction, except the area in proximity with the domain walls, as shown in Fig. 3b, and in Fig. 3d due to the shear strain induced by $71^{\circ}$ domain walls ${ }^{28}$. However, this shear strain domain wall is not significant in the $109^{\circ}$ case, as shown in Fig. 3c. This extra strain perturbation can distort the structure of the YBCO, resulting in a suppression of the $T_{\mathrm{c}}$ near the domain walls. This is consistent with the RSM result in Fig. 1f, where the YBCO is partially relaxed due to the lattice mismatch. Similar to the argument for the $109^{\circ}$ case, the parallel geometry shows a higher $T_{\mathrm{c}}$ than that of the perpendicular geometry. Thus, in both the $109^{\circ}$ and $71^{\circ}$ cases, an anisotropy in the superconductivity is very expected and needs to be further investigated by a spatially resolved characterization technique.

To reveal the mechanism of the anisotropic superconducting behavior, XSTM was employed to study the local electronic structure across the interfaces of the heterostructures. Both the $109^{\circ}$ and $71^{\circ}$ cases were investigated at $25 \mathrm{~K}$. The geometry of the XSTM measurement is schematically illustrated in Fig. 4a. All samples were cleaved in an ultrahigh vacuum chamber to obtain a clean cross-section morphology, as shown in Fig. $4 \mathrm{~b}$, and then transferred to the XSTM chamber. The protection of the YBCO layer from moisture and the STM tip can be assisted by the deposition of a conducting layer, namely, AZO, on top of the YBCO layer. The experimental details of the XSTM measurements can be found in the Supplementary Materials (S8 and S9) and Materials and methods section. To explore the local electronic structures in the cross-sections of the $\mathrm{YBCO} / \mathrm{BFO}$ heterostructures, the locations of the domains and domain walls $\left(71^{\circ}\right.$ and $\left.109^{\circ}\right)$ in the BFO layer were mapped first. 


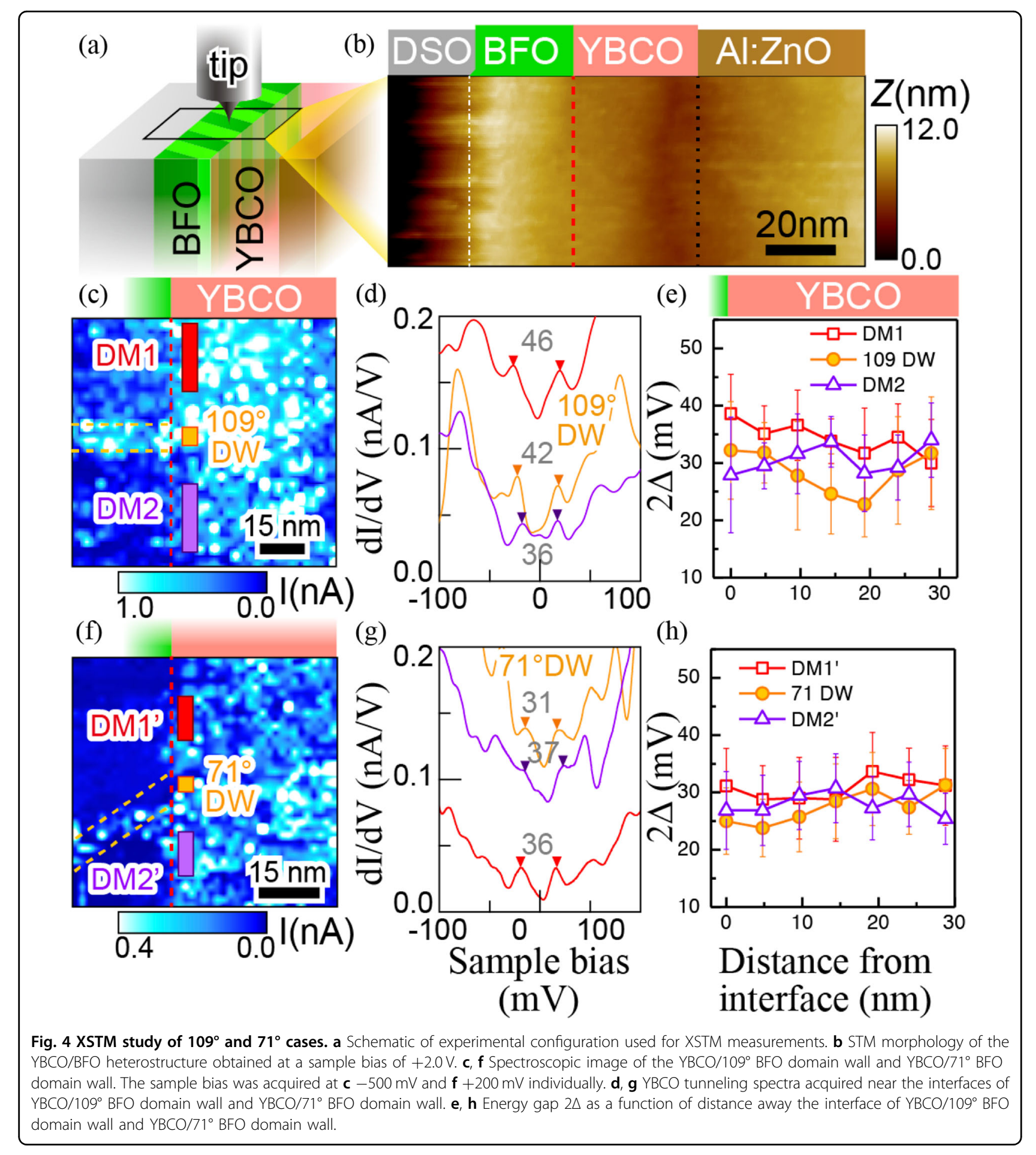

Based on our previous STM study of BFO domain walls ${ }^{29}$, the locations of the $71^{\circ}$ and $109^{\circ}$ domain walls can be determined from the mapping of the tunneling conductance (Fig. 4c, f), where the yellow dotted line highlights the edges of the $109^{\circ}\left(71^{\circ}\right)$ domain wall and the red dotted line indicates the interface between the YBCO and BFO layers. With this map, we can then investigate the electronic structures in the YBCO layer at the nanoscale. The detailed electronic structures of the YBCO layer were explored in three locations in each sample individually: the areas adjacent to the BFO domains (marked by red and purple rectangles (denoted as $\mathrm{DM} 1^{\prime}$ and $\left.\mathrm{DM} 2^{\prime}\right)$ ) and the area adjacent to the BFO domain wall (marked by an orange rectangle (denoted as DW)). The sizes of the 
energy gap of the YBCO at these locations can be estimated from the differential $I-V(\mathrm{~d} I / \mathrm{d} V)$ curves shown in Fig. $4 \mathrm{~d}, \mathrm{~g}^{30}$. Interestingly, the energy gaps extracted at the three locations show an obvious difference in size: they were 46,42 , and $36 \mathrm{mV}$ for DM1, DW, and DM2, respectively, in the $109^{\circ}$ case; and they were 36,31 and $37 \mathrm{mV}$ for $\mathrm{DM}^{\prime}, \mathrm{DW}$, and $\mathrm{DM} 2^{\prime}$, respectively, in the $71^{\circ}$ case. It is very important to note that the size of the YBCO energy gap measured adjacent to the DW area is between that of the two domains in the $109^{\circ}$ case, while the sizes of the YBCO energy gap measured adjacent to the domain areas in the $71^{\circ}$ case have very similar values, and the energy gap at $D W$ is smaller than that for both of them. The variation in the energy gap size suggests different $T_{\mathrm{c}}$ values in these regions ${ }^{31,32}$. These results support the anisotropic superconductivity found in the transport and can be well explained by the ferroelectric field and strain effects induced by the BFO domain patterns during the YBCO growth.

In the $109^{\circ}$ case, the neighboring domains in the $\mathrm{BFO}$ layer have opposite polarization components along the out-of-plane direction, which are pointing away from and toward the $\mathrm{YBCO}$ layer. Here, we argue that during $\mathrm{YBCO}$ growth, the periodical electrostatic boundary condition caused a redistribution of oxygen because BFO has a high Curie temperature $(\sim 1100 \mathrm{~K})$, where the ferroelectric domain formed during deposition, leading to a modulation of the YBCO doping levels. As a consequence, two different sizes of energy gaps, 46 and $36 \mathrm{mV}$, are observed (Fig. 4d). The polarization directions of the BFO domains corresponding to the DM1 and DM2 areas can also be determined to be pointing away from $\mathrm{YBCO}$ for $\mathrm{DM} 1$ and pointing toward YBCO for DM2 based on the underdoped YBCO layer. As the transition area between the domains, the domain wall exhibits a negligible out-ofplane component for the ferroelectric polarization ${ }^{33,34}$. Thus, the size of the energy gap measured in the DW region falls between the DM1 and DM2 regions. As the measured spectra were acquired away from the $\mathrm{YBCO} /$ $\mathrm{BFO}$ interface, the difference between the energy gap sizes of the DM1 and DM2 regions diminishes $\sim 28 \mathrm{~nm}$ away from the interface (Fig. 4e). Based on these results, the YBCO regions in the domains with different out-of-plane ferroelectric components correspond to two different $T_{\mathrm{C}} \mathrm{S}$, resulting in anisotropic superconductivity. On the other hand, it is a different story for the $71^{\circ}$ case. The neighboring domains in the BFO layer have the same polarization components along the out-of-plane direction, both pointing away from the YBCO layer, which leads to the same doping level in the YBCO regions in the DM1' and DM2' areas. Thus, the strain effect becomes the dominant mechanism and causes the difference in the size of the energy gap between the $\mathrm{DM}^{\prime}$ and the $\mathrm{DW}$ regions. A similar relaxation effect can also be observed with a decreased relaxation length of $\sim 21 \mathrm{~nm}$ (Fig. $4 \mathrm{~h}$ ). In this case, the anisotropic superconductivity is very likely to be induced by the domain walls. These results are in good agreement with the results predicted by the phase-field simulations. In both cases, the periodic domain patterns caused periodic modulations of inhomogeneity (nanoscale phase separation of $\mathrm{YBCO}$ ), which directly led to the anisotropic superconductivity.

\section{Conclusion}

In summary, we successfully demonstrated an artificially created anisotropic superconductivity induced by the periodic domain pattern in $\mathrm{BFO}$ with $\mathrm{YBCO} / \mathrm{BFO}$ heterostructures. The anisotropic transport behavior can be observed in both the $109^{\circ}$ and $71^{\circ}$ cases with the superconducting easy axis always along the domain wall direction in the BFO. Two mechanisms-the strain effect and the ferroelectric field effect during YBCO growthlead to this anisotropic superconductivity. The formation of nanoscale phase separation based on an intimate contact between the superconductor and multiferroic suggests that new device architectures, such as a selfassembled Josephson junction (superconductor/normal metal/superconductor), can be achieved and ultimately lead to new multifunctional devices.

\section{Acknowledgements}

This work is supported by the Ministry of Science and Technology of Republic of China (Grant Nos. MOST 106-2119-M-009-011-MY3, 106-2628-E-009-001MY2, 106-2218-E-009-021, 106-2923-M-009-003-MY2, 107-2112-M-001-042MY3, 107-2218-E-007-050). Prof. Y.P. Chiu acknowledges the support from the Ministry of Science and Technology of Republic of China (under contract no. MOST 106-2628-M-002-011-MY3, MOST108-2633-M-001-001). The work at Penn State was supported by the US Department of Energy (DOE), Office of Basic Energy Sciences, Division of Materials Sciences and Engineering under Award FG02-07ER46417 (F.X. and L.-Q.C.).

\section{Authors' contributions \\ Y.-L.H and Y.-H.C. designed the experiments. Y.-L.H carried out materials synthesis, characterization, and electrical measurements. J.-C.L. conducted electrical measurements. B.-C.H. and Y.-T.W. conducted XSTM measurements. F.X. carried phase-field simulations. R.H. performed HR-TEM imaging. H.J.L. performed HR-XRD measurements. Y.-L.H and Y.-H.C. wrote the paper. All authors made contributions to writing the manuscript. The work was conceived and guided by Y.-H.C. All authors discussed the results and commented on the manuscript.}

\section{Author details}

'Department of Materials Science and Engineering, National Chiao Tung University, Hsinchu, Taiwan. ${ }^{2}$ Institute of Physics, Academia Sinica, Taipei, Taiwan. ${ }^{3}$ Department of Physics, National Taiwan Normal University, Taipei, Taiwan. ${ }^{4}$ Department of Materials Science and Engineering, The Pennsylvania State University, University Park, Pennsylvania, USA. ${ }^{5}$ Department of Materials Science and Engineering, National Chung Hsing University, Taichung, Taiwan. ${ }^{6}$ Key Laboratory of Polar Materials and Devices, Ministry of Education, East China Normal University, Shanghai, China. ${ }^{7}$ Nanostructures Research Laboratory, Japan Fine Ceramics Center, Nagoya, Japan. ${ }^{8}$ Institute of Physics, National Chiao Tung University, Hsinchu, Taiwan. ${ }^{9}$ Department of Physics, National Taiwan University, Taipei, Taiwan. ${ }^{10} \mathrm{Center}$ for Emergent Functional Matter Science, National Chiao Tung University, Hsinchu, Taiwan 


\section{Conflict of interest}

The authors declare that they have no conflict of interest.

\section{Publisher's note}

Springer Nature remains neutral with regard to jurisdictional claims in published maps and institutional affiliations.

Supplementary information is available for this paper at https://doi.org/ 10.1038/s41427-019-0178-y.

Received: 16 July 2019 Revised: 5 September 2019 Accepted: 16 September 2019.

Published online: 6 December 2019

\section{References}

1. Spaldin, N. A. \& Fiebig, M. The renaissance of magnetoelectric multiferroics. Science 309, 391 (2005).

2. Ramesh, R. \& Spaldin, N. A. Multiferroics: progress and prospects in thin films. Nat. Mater. 6, 21 (2007).

3. Chu, Y.H., Martin, L. W., Martin, Holcomb, M. B. \& Ramesh, R. Controlling magnetism with multiferroics. Mater. Today 10, 16 (2007).

4. Fiebig, M., Lottermoser, T., Meier, D. \& Trassin, M. The evolution of multiferroics, Nat. Rev. Mater. 8, 16046 (2016).

5. Spaldin, N. A. \& Ramesh, R. Advances in magnetoelectric multiferroics. Nat. Mater. 18, 203 (2019).

6. Wang, J. et al. Epitaxial $\mathrm{BiFeO}_{3}$ multiferroic thin film heterostructures. Science 299, 1719 (2003).

7. Teague, J. R., Gerson, R. \& James, W. J. Dielectric hysteresis in single crystal $\mathrm{BiFeO}_{3}$. Solid State Commun. 8, 1073 (1970).

8. Smolenskii, G. A. \& Chupis, I. Ferroelectromagnets. Sov. Phys. Usp. 25, 475 (1982).

9. Manipatruni, S. et al. Scalable energy-efficient magnetoelectric spin-orbit logic. Nature 565, 7737 (2019).

10. Merz, J. W. Domain formation and domain wall motions in ferroelectric $\mathrm{BaTiO}_{3}$ single crystals. Phys. Rev. 95, 690 (1954).

11. $\mathrm{He}, \mathrm{Q}$. et al. Magnetotransport at domain walls in $\mathrm{BiFeO}_{3}$. Phys. Rev. Lett. 108, 067203 (2012)

12. Martin, W. L. et al. Nanoscale control of exchange bias with $\mathrm{BiFeO}_{3}$ thin films. Nano Lett. 8, 2050 (2008).

13. Heron, J. T. et al. Electric-field-induced magnetization reversal in a ferromagnet-multiferroic heterostructure. Phys. Rev. Lett. 107, 217202 (2011).

14. Heron, J. T. et al. Deterministic switching of ferromagnetism at room temperature using an electric field. Nature 516, 370-373 (2014).

15. Trassin, M. et al. Interfacial coupling in multiferroic/ferromagnet heterostructures. Phys. Rev. B 87, 134426 (2013).
16. $\mathrm{You}, \mathrm{L}$. et al. Origin of the uniaxial magnetic anisotropy in $\mathrm{La}_{0.7} \mathrm{Sr}_{0.3} \mathrm{MnO}_{3}$ on stripe-domain $\mathrm{BiFeO}_{3}$. Phys. Rev. B 88, 184426 (2013).

17. Ju, C. et al. Anomalous electronic anisotropy triggered by ferroelastic coupling in multiferroic heterostructures. Adv. Mater. 28, 876 (2016).

18. Streiffer, S. K. et al. Domain patterns in epitaxial rhombohedral ferroelectric films. I. geometry and experiments. J. Appl. Phys. 83, 2742 (1998).

19. Chu, Y.H. et al. Domain control in multiferroic $\mathrm{BiFeO}_{3}$ through substrate vicinality. Adv. Mater. 19, 2662 (2007).

20. Chu, Y.-H. et al. Nanoscale control of domain architectures in $\mathrm{BiFeO}_{3}$ thin films. Nano Lett. 9, 1726 (2009).

21. Floquet, N. \& Valot, C. Ferroelectric domain walls in $\mathrm{BaTiO}_{3}$ : structural wall model interpreting fingerprints in XRPD diagrams. Ferroelectrics 234, 107 (1999).

22. Ando, Y., Segawa, K., Komiya, S. \& Lavrov, A. N. Electrical resistivity anisotropy from self-organized one dimensionality in high-temperature superconductors. Phys. Rev. Lett. 88, 137005 (2002). 2002.

23. Hilgenkamp, H. \& Mannhart, J. Grain boundaries in high-T $T_{c}$ superconductors. Rev. Mod. Phys. 74, 485 (2002).

24. Tolpygo, S. K. Lin, J.Y., Gurvitch, M., Hou, S. Y. \& Phillips, J. M. Effect of oxygen defects on transport properties and $\mathrm{T}_{c}$ of $\mathrm{YBa}_{2} \mathrm{Cu}_{3} \mathrm{O}_{6}+x$ : displacement energy for plane and chain oxygen and implications for irradiation-induced resistivity and $T_{C}$ suppression. Phys. Rev. B 53, 12462 (1996).

25. Tolpygo, S. K., Lin, J.-Y., Gunvitch, M., Hou, S. Y. \& Phillips, J. M. Universal suppression by in-plane defects in high-temperature superconductors: implications for pairing symmetry. Phys. Rev. B 53, 12454 (1996).

26. Ahn, C. H. et al. Electrostatic modulation of superconductivity in ultrathin $\mathrm{GdBa}_{2} \mathrm{Cu}_{3} \mathrm{O}_{7-x}$ films. Science 284, 1152 (1999).

27. Crassous, A. et al. Nanoscale electrostatic manipulation of magnetic flux quanta in ferroelectric/superconductor $\mathrm{BiFeO}_{3} / \mathrm{YBa}_{2} \mathrm{Cu}_{3} \mathrm{O}_{7}-\delta$ heterostructures. Phys. Rev. Lett. 107, 247002 (2011).

28. Li, L. et al. Control of domain structures in multiferroic thin films through defect engineering. Adv. Mater. 30, 1802737 (2018).

29. Chiu, Y.P. et al. Atomic-scale evolution of local electronic structure across multiferroic domain walls. Adv. Mater. 23, 1530 (2011).

30. Edwards, H. L., Markert, J. T. \& Lozanne, A. L. Energy gap and surface structure of $\mathrm{YBa}_{2} \mathrm{Cu}_{3} \mathrm{O}_{7-x}$ probed by scanning tunneling microscopy. Phys. Rev. Lett. 69 , 2967 (1992).

31. Hainzl, C. \& Seiringer, R. Critical temperature and energy gap for the BCS equation. Phys. Rev. B 77, 184517 (2008).

32. $\mathrm{Ge}, \mathrm{J}$. F. et al. Phase diagram and electronic indication of high-temperature superconductivity at $65 \mathrm{~K}$ in single-layer FeSe films. Nat. Mater. 12, 605 (2013).

33. Lubk, A., Gemming, S. \& Spaldin, N. A. First-principles study of ferroelectric domain walls in multiferroic bismuth ferrite. Phys. Rev. B 80, 104110 (2009).

34. Wang, $\mathrm{Y}$. et al. $\mathrm{BiFeO}_{3}$ domain wall energies and structures: a combined experimental and density functional theory $+U$ study. Phys. Rev. Lett. 110 267601 (2013). 